OPEN ACCESS

Edited by: Antonio Colantuoni, University of Naples Federico II, Italy

Reviewed by:

Stefano Tarantini, University of Oklahoma Health Sciences Center, United States Dominga Lapi, University of Naples Federico II, Italy

*Correspondence: Martin Müller martin.mueller@/uks.ch

Specialty section: This article was submitted to Vascular Physiology, a section of the journal Frontiers in Physiology

Received: 19 July 2019 Accepted: 11 October 2019 Published: 24 October 2019

Citation:

Müller M and Österreich M (2019) Cerebral Microcirculatory Blood Flow

Dynamics During Rest and a Continuous Motor Task. Front. Physiol. 10:1355. doi: 10.3389/fphys.2019.01355

\section{Cerebral Microcirculatory Blood Flow Dynamics During Rest and a Continuous Motor Task}

\author{
Martin Müller* and Mareike Österreich \\ Neurovascular Laboratory, Neurocenter, Lucerne Kantonsspital, Lucerne, Switzerland
}

Objectives: To examine the brain's microcirculatory response over the course of a continuous 5-min elbow movement task in order to estimate its potential role in grading vaso-neural coupling compared to the macrocirculatory response.

Methods: We simultaneously recorded cerebral blood flow velocity (CBFV), changes in oxygenated/deoxygenated hemoglobin concentrations ([oxHb], [deoxHb]), blood pressure (BP), and end-tidal $\mathrm{CO}_{2}$ over 5 -min periods of rest and left elbow movements in 24 healthy persons (13 women and 11 men of mean age \pm SD, $38 \pm 11$ years). A low frequency range $(0.07-0.15 \mathrm{~Hz})$ was used for analysis by transfer function estimates of phase and gain.

Results: Elbow movement led to a small BP increase (mean BP at rest $83 \mathrm{~mm} \mathrm{Hg}$, at movement 87; $p<0.01$ ) and a small $\mathrm{ETCO}_{2}$ decrease (at rest $44.6 \mathrm{~mm} \mathrm{Hg}$, at movement $41.7 \mathrm{~mm} \mathrm{Hg} ; p<0.01$ ). Further, it increased BP-[oxHb] phase from $55^{\circ}$ (both sides) to $74^{\circ}$ (right; $p<0.05$ ) $/ 69^{\circ}$ (left; $p<0.05$ ), and BP-[deoxHb] phase from $264^{\circ}$ (right) $/ 270^{\circ}$ (left) to $288^{\circ}$ (right; $p<0.05$ ) $/ 297^{\circ}$ (left; $p=0.09$ ). The cerebral mean transit time at $0.1 \mathrm{~Hz}$ of $5.6 \mathrm{~s}$ of rest remained unchanged by movement. Elbow movement significantly decreased BP-CBFV gain on both sides, and BP-CBFV phase only on the right side ( $p=0.05)$.

Conclusion: Elbow movement leads to an increased time delay between BP and [oxHb]/ [deoxHb] while leaving the cerebral mean transit time unchanged. Phase shifting is usually the more robust parameter when using a transfer function to estimate dynamic cerebral autoregulation; phase shifting at the microcirculatory level seems to be a better marker of VNC-induced changes than phase shifting between BP and CBFV.

\footnotetext{
Keywords: cerebral autoregulation, cerebral microcirculation, motor task, evoked flow, near-infrared spectroscopy, transcranial Doppler
}

\section{INTRODUCTION}

Considering its pathophysiologic relevance to high blood pressure (BP), microangiopathic diseases, mild cognitive impairment, and dementia, the examination of cerebral microcirculation has been gaining widespread research interest (Birns et al., 2009; Pantoni, 2010; Marmarelis et al., 2014, 2017; Duncombe et al., 2017; de Heus et al., 2018; Müller et al., 2019). In addition to morphological changes, as demonstrated by computerized tomography or magnetic resonance imaging (MRI), regulation of the microcirculatory blood supply is believed to be a relevant part of disease progression (Birns et al., 2009; Pantoni, 2010; Duncombe et al., 2017; Müller et al., 2019). 
Near-infrared spectroscopy (NIRS) offers the opportunity to observe oxygenated and deoxygenated hemoglobin concentration changes ([oxHb], [deoxHb]) in the microcirculation of the brain's upper cortical layer with a relatively high temporal resolution (Murkin and Arango, 2009). Their dynamic interplay with BP or cerebral blood flow velocity (CBFV) may potentially represent an early disease marker. While much research has been carried out on the interplay between BP and transcranial Dopplerderived CBFV for assessing cerebrovascular dynamics (for a comprehensive overview, see Claassen et al., 2016), the usefulness of NIRS parameters as a substitute for CBFV in this context has, surprisingly, not yet been well evaluated.

Cerebral blood flow (CBF) is the combined result of $\mathrm{BP}$, metabolic, and neuronal regulatory influences. In recent years, the influence of vaso-neural coupling (VNC) on CBF was determined to be a relevant contributor which can be disturbed even when cerebral autoregulation remains intact (Salinet et al., 2013, 2015; Caldas et al., 2018). To assess the dynamics of CA (dCA), applying a transfer function analysis (TFA) on the relationship between $\mathrm{BP}$ and $\mathrm{CBFV}$ is the most frequently used approach nowadays. When applied over 5-min recording periods, the phase and gain results are reliable, and phase is more robust than gain for the grading of CA capabilities (Claassen et al., 2016; Sanders et al., 2018). Most VNC studies used short task periods $(\leq 60$ s) to investigate VNC (Chance et al., 1993; Obrig et al., 2000; Salinet et al., 2013, 2015; Caldas et al., 2018), and are, therefore, unsuitable for assessing TFA (Claassen et al., 2016). In addition to the TFA of the BP-CBFV macrocirculatory relationship, the TF analyses of the dynamics of the interaction between $\mathrm{BP}$ and $[\mathrm{oxHb}]$, as well as of [deoxHb] (microcirculatory response), have also shown that it is possible to grade CA failure (Reinhard et al., 2006; Cheng et al., 2012; Elting et al., 2018). The motivation behind and the hypothesis of our present study is that a long-lasting stimulus in a normal CA state shows a TFA response (either in the macrocirculatory or in the microcirculatory response) that can be used to grade VNC. Since the final aim of our study is to assess whether alterations in this VNC response interfere with patient's daily life activities or rehabilitation capabilities, further studies on patients using this setup could not be justified if this hypothesis were to turn out not to be true.

\section{MATERIALS AND METHODS}

The study was approved by the Ethics Committee of Northwest and Central Switzerland and abided by the tenets of the Declaration of Helsinki, using good standards of clinical practice. Written informed consent was obtained from all subjects $[n=24 ; 13$ women, 11 men; mean age \pm SD (standard deviation): $38 \pm 11$ years (range $24-62$ years)].

\section{Experimental Setting and Instrumentation}

All investigations were performed in the late morning with subjects in a supine position with their head elevated at approximately $30^{\circ}$ in a dimly lit room. Coffee or tea was last ingested a minimum of $4 \mathrm{~h}$ before the beginning of the assessments. Subjects were mainly right-handed (20 of 25) and all were non-smokers. Participants were carefully instructed to flex and extend their elbow over the full range of movement (= one movement cycle) at a pace of $1 \mathrm{~Hz}$, as signaled by the beep of a computerized metronome. Before mounting the probes, subjects practiced performing several elbow movement cycles according to the metronome's pacing.

We assessed microcirculation using the NIRO-200NX NIRS device (Hamamatsu Photonics, Herrsching, Germany). This NIRS device emits infrared light at three frequencies (735, 810 , and $850 \mathrm{~nm}$ ). After absorption by oxygenated and deoxygenated hemoglobin, the backscattered light has a different intensity. The differences in light intensities between the emitted and backscattered lights are correlated with the concentration of oxygenated and deoxygenated hemoglobin in the brain's upper layers. We used self-adhesive NIRS probes in which the light emitting diode (LED) and the detecting photodiode were fixed, separated by a distance of $4 \mathrm{~cm}$. The detecting probe was placed at the lateral frontotemporal skull to ensure it was above the vascular territory of the middle cerebral artery (MCA), and the emitting probe was placed $4 \mathrm{~cm}$ away at the frontal skull. After initial adjustments to determine the baseline hemoglobin concentration (in $\mu \mathrm{mol} / \mathrm{L}$ ), the NIRS device provides continuous percentage changes in [oxHb] and [deoxHb].

Although we mainly focused on NIRS, because the BP-CBFV system is a more well-established method of assessing cerebrovascular dynamics, and because we wanted to interpret the BP-[oxHb] and BP-[deoxHb] findings in light of the best established method, we also recorded CBFV. After fixing the NIRS probes, a head holder provided by the manufacturer of the TCD device (MultidopX, DWL; Compumedics, Sipplingen, Germany) was placed for the TCD examination. Both MCAs were identified using common criteria, and the TCD probes $(2 \mathrm{MHz})$ were positioned with the help of the head holder. End-tidal $\mathrm{CO}_{2}\left(\mathrm{ETCO}_{2}\right)$ was measured via a small nostril tube connected to a capnograph embedded in the TCD device. BP was measured by finger plethysmography (Finometer Pro; Finapres Medical Systems, Amsterdam, The Netherlands) placed at the fingertip of the right index finger, with special attention paid to calibrating it to the pressure of the brachial artery. The BP signal was imported into the TCD device and simultaneously recorded along with $\mathrm{CBFV}$ and $\mathrm{ETCO}_{2}$.

After all probes were placed and the subject familiarized him- or herself with the surroundings and experimental setting, we started with a 5 -min baseline recording period. Subsequently, the left elbow movement was performed at the $1-\mathrm{Hz}$ frequency set by the computerized metronome. The BP measurement instrument was placed on the fingertip of the right index finger such that the right arm could not be moved.

\section{Data Preparation and Analysis}

$\mathrm{CBFV}, \mathrm{BP}$, and $\mathrm{ETCO}_{2}$ data were collected at $100 \mathrm{~Hz}$ and NIRS data were collected at $5 \mathrm{~Hz}$. The data were analyzed using Matlab (2018b, Math Works Inc., Natick, MA, USA). The data were visually inspected for artifacts, and only artifactfree 5-min periods of data were used. After aligning the time series with their common starting time point, each raw data time series was resampled by averaging over a 1-s interval. 
These new data segments then underwent transfer function analysis (TFA). The coherence and TFA estimates of phase $\left(\right.$ in $\left.^{\circ}\right)$ and gain between the different time series were extracted from their respective power auto spectra or cross spectra using Welch's averaged periodogram method with a Hann window length of $100 \mathrm{~s}$, a window overlap of 50\%, and a total Fast Fourier Transformation data length of $300 \mathrm{~s}$, thereby allowing for calculations over a frequency range of $0.02-0.40 \mathrm{~Hz}$.

Because CA is mostly regulated in the frequency range of $0.07-0.15 \mathrm{~Hz}$, and coherence has been shown to be low in the frequency range of $0.02-0.07$ when [oxHb] and [deoxHb] are involved (Müller et al., 2016), we decided to only use the $0.07-0.15-\mathrm{Hz}$ frequency range for this analysis. To calculate means, we averaged phase and gain values with a coherence $\geq 0.34$ (Claassen et al., 2016). When large phase wrap-arounds were present in the phase spectrum plots, we subsequently corrected phase by adding or subtracting $360^{\circ}$ (as recommended in Claassen et al., 2016). All analyses were primarily performed using the phase spectrum. When necessary for comparing with previously published literature, phases were transformed to their time domains. Cerebral mean transit times were calculated as phase (BP-[deoxHb] $)$ - phase (BP-[oxHb]).

For each period, we also calculated cerebrovascular resistance as mean $\mathrm{BP} /$ mean $\mathrm{CBFV}$, in addition to the heart rate derived from the BP signal.

\section{Statistical Analysis}

The Matlab Statistical Toolbox was used for all data analyses. Using a Kolmogorov-Smirnov test, all data were shown to have a normal distribution, and the data are reported as mean \pm standard deviation (SD). To compare means between the two periods (baseline and elbow movement), we used a one-way ANOVA, including, when indicated, a correction for multiple comparisons. A regression analysis was used to test whether the results were dependent on $\mathrm{ETCO}_{2}$, BP variability (as calculated as the $\mathrm{SD}$ of $\mathrm{BP}$ ), cerebrovascular resistance (BP/CBFV), or BP-derived heart rate. Values of $p \leq 0.05$ were considered statistically significant.

\section{RESULTS}

Artifact-free recordings were acquired bilaterally in all subjects. The hemodynamic baseline characteristics, including $\mathrm{ETCO}_{2}$, are reported in Table 1. Elbow movement induced significant increases in $\mathrm{BP}$, heart rate, $\mathrm{CBFV}$, [oxHB], and CVR (on the right side only), and a significant decrease in $\mathrm{ETCO}_{2}$ and [deoxHb].

The time course of the group average means of $\mathrm{BP}, \mathrm{ETCO}_{2}$, $\mathrm{CBFV},[\mathrm{oxHb}]$, and [deoxHb] is shown in Figure 1. Changes in CBFV, [oxHb], and [deoxHb] start very shortly after beginning the motor task. $\mathrm{ETCO}_{2}$ decreased $5 \mathrm{~s}$ thereafter, and, of note, BP rose afterward, after about $20 \mathrm{~s}$. Subsequently, all variables remained largely constant until the end of the stimulus period. Mean $[\mathrm{oxHb}]$ changes over the total stimulus period were significantly higher in the right compared to left side $(p<0.001)$.

TFA results (Table 2) demonstrate, under resting conditions, a phase lead of $\mathrm{CBFV}$ to $\mathrm{BP}\left(\right.$ mean $\left.\approx-36^{\circ}\right)$ and a time lag
TABLE 1 | Hemodynamic baseline variables (mean \pm standard deviation) at rest and during elbow movement over 5 -min periods at a frequency of $1 \mathrm{~Hz}$.

\begin{tabular}{lccc}
\hline & Baseline & Elbow movement & ANOVA \\
\cline { 1 - 3 } Mean BP $(\mathrm{mm} \mathrm{HG})$ & $83 \pm 13$ & $87 \pm 17$ & $<0.01$ \\
BP variability (mm HG) & $16 \pm 2$ & $17 \pm 2$ & $\mathrm{~ns}$ \\
Mean ETCO $(\mathrm{mm} \mathrm{HG})$ & $44.6 \pm 3.0$ & $41.7 \pm 3.4$ & $<0.01$ \\
Mean heart rate (beats/min) & $65 \pm 9$ & $74 \pm 11$ & $<0.01$ \\
Mean CBFV right (cm/s) & $65 \pm 12$ & $69 \pm 12$ & $<0.01$ \\
Mean CBFV left (cm/s) & $64 \pm 11$ & $70 \pm 13$ & $<0.01$ \\
Mean CVR right & $1.35 \pm 0.29$ & $1.28 \pm 0.28$ & $<0.01$ \\
Mean CVR left & $1.32 \pm 0.31$ & $1.31 \pm 0.32$ & $\mathrm{~ns}$ \\
Mean [oxHb]r (\%) & $0.08 \pm 0.11$ & $0.55 \pm 0.11$ & $<0.001$ \\
Mean [oxHb]l (\%) & $-0.001 \pm 0.08$ & $0.19 \pm 0.09$ & $<0.001$ \\
Mean [deoxHb]r (\%) & $-0.56 \pm 0.04$ & $-0.81 \pm 0.11$ & $<0.001$ \\
Mean [deoxHb]l (\%) & $-0.60 \pm 0.05$ & $-0.84 \pm 0.07$ & $<0.001$ \\
\hline
\end{tabular}

$B P$, blood pressure; $\mathrm{ETCO}_{2}$, end-tidal carbon dioxide; $\mathrm{CBFV}$, cerebral blood flow velocity; [oxHb], oxygenated hemoglobin; [deoxHb], deoxygenated hemoglobin; r, right; l, left.

TABLE 2 | Transfer function estimates (phase and gain) for blood pressure and cerebral blood flow velocity-dependent hemodynamic pairs (mean \pm standard deviation).

\begin{tabular}{|c|c|c|c|}
\hline & Baseline & Elbow movement & ANOVA \\
\hline \multicolumn{4}{|c|}{ BP-dependent system } \\
\hline \multicolumn{4}{|l|}{ Phase (in degrees) } \\
\hline $\mathrm{BP}-\mathrm{CBFV} r$ & $-36 \pm 10$ & $-43 \pm 9$ & $p=0.05$ \\
\hline BP-CBFVI & $-37 \pm 13$ & $-40 \pm 17$ & ns \\
\hline BP-[oxHb]r & $55 \pm 27$ & $76 \pm 34$ & $p<0.05$ \\
\hline BP-[oxHb]l & $55 \pm 28$ & $69 \pm 31$ & $p<0.05$ \\
\hline BP-[deoxHb]r & $264 \pm 38$ & $288 \pm 31$ & $p<0.05$ \\
\hline BP-[deoxHb]l & $270 \pm 47$ & $297 \pm 33$ & $p=0.09$ \\
\hline \multicolumn{4}{|c|}{ Gain (in \%/mm Hg) } \\
\hline $\mathrm{BP}-\mathrm{CBFV} r$ & $0.97 \pm 0.35$ & $0.73 \pm 0.38$ & $p<0.01$ \\
\hline BP CBFVI & $1.01 \pm 0.44$ & $0.76 \pm 0.31$ & $p<0.01$ \\
\hline $\mathrm{BP}-[\mathrm{oxHbr}]$ & $0.005 \pm 0.01$ & $0.002 \pm 0.01$ & ns \\
\hline $\mathrm{BP}-[\mathrm{oxHb}] \mathrm{I}$ & $0.007 \pm 0.01$ & $0.007 \pm 0.03$ & ns \\
\hline BP-[deoxHb]r & $-0.003 \pm 0.005$ & $-0.001 \pm 0.007$ & ns \\
\hline BP-[deoxHb]l & $-0.003 \pm 0.005$ & $-0.002 \pm 0.01$ & ns \\
\hline \multicolumn{4}{|c|}{ CBFV-dependent system } \\
\hline \multicolumn{4}{|c|}{ Phase (in degrees) } \\
\hline CBVF-[OxHb]r & $47 \pm 27$ & $61 \pm 23$ & $p=0.06$ \\
\hline CBFV-[oxHb]l & $46 \pm 25$ & $52 \pm 35$ & ns \\
\hline CBFV-[deoxHb]r & $279 \pm 41$ & $261 \pm 48$ & ns \\
\hline CBFV-[deoxHb]l & $273 \pm 20$ & $269 \pm 37$ & ns \\
\hline \multicolumn{4}{|l|}{ Gain (in \%/cm/s) } \\
\hline CBFV-[oxHb]r & $0.007 \pm 0.03$ & $0.009 \pm 0.01$ & ns \\
\hline CBFV-[oxHb]l & $0.002 \pm 0.04$ & $0.008 \pm 0.03$ & ns \\
\hline CBFV-[deoxHb]r & $-0.003 \pm 0.02$ & $-0.002 \pm 0.01$ & ns \\
\hline CBFV-[deoxHb]l & $-0.009 \pm 0.03$ & $-0.004 \pm 0.02$ & ns \\
\hline \multicolumn{4}{|c|}{ Mean transit time (in degrees) } \\
\hline Right & $209 \pm 49$ & $216 \pm 33$ & ns \\
\hline Left & $201 \pm 46$ & $226 \pm 53$ & ns \\
\hline
\end{tabular}

$B P$, blood pressure; $C B F V$, cerebral blood flow velocity; [oxHb], oxygenated hemoglobin concentration; [deoxHb], deoxygenated hemoglobin concentration; r, right; I, left; mean transit time calculated as $(\mathrm{BP}-[\mathrm{HH} \mathrm{H}])-\left(\mathrm{BP}-\left[\mathrm{O}_{2} \mathrm{Hb}\right]\right)$.

of [oxHb] $\left(\approx 55^{\circ}\right)$ and [deoxHb] $\left(\approx 266^{\circ}\right)$ behind BP. [oxHb] was $44^{\circ}$ behind $\mathrm{CBFV}$, and [deoxHb] $275^{\circ}$ behind CBFV.

Elbow movement induced several changes, among which the phase changes are summarized in Figure 2. First, elbow movement reduced the mean gain between $\mathrm{BP}$ and $\mathrm{CBFV}$ on both sides significantly from $\approx 1$ to $\approx 0.75 \% / \mathrm{mmHg}$. The increases in the 



FIGURE 1 | Time course of (A) blood pressure (BP), cerebral blood flow velocity (CBFV), end-tidal carbon dioxide (ETCO $)_{2}$, (B) concentration of oxygenated ([oxHb]) and deoxygenated ([deoxHb]) hemoglobin during baseline at rest and elbow movement time periods. BP, blood pressure; CBFV, cerebral blood flow velocity; $\mathrm{ETCO}_{2}$, end-tidal carbon dioxide; [oxHb], oxygenated hemoglobin; [deoxHb], deoxygenated hemoglobin. Bars indicate the standard deviation over all values of each time period. For convenience, since the SD bars of CBFV are nearly identical for both sides, we decided to only show one for both sides. The thick gray bar above the $\mathrm{X}$-axis represents the timing of the elbow movement.

negative mean phase between $\mathrm{BP}$ and $\mathrm{CBFV}$ (from $-36^{\circ}$ (right) $/-37^{\circ}$ (left) to $-40^{\circ} /-43^{\circ}$; right n.s., left $p=0.05$ ) were small. Second, elbow movement increased the positive mean phase shift between $\mathrm{BP}$ and $[\mathrm{oxHb}]$ from $55^{\circ}$ (both sides) to $74^{\circ}$ (right; $\left.p<0.05\right) / 69^{\circ}$ (left; $p<0.05$ ), and between BP and [deoxHb] from $264^{\circ}$ (right) $/ 270^{\circ}$ (left) to $288^{\circ}$ (right; $p<0.05$ ) $/ 297^{\circ}$ (left; $p=0.09$ ). The mean transit time was not different between rest and elbow movement. Third, elbow movement increased the positive mean phase shift between CBFV and [oxHb] on both sides (from $47^{\circ}$ to $62^{\circ}$ on the right side, from $48^{\circ}$ to $52^{\circ}$ on the left side), which was relevant by trend $(p=0.06)$ on the right side.
On regression analysis, no TFA parameter was found to be related to heart rate, $\mathrm{ETCO}_{2}, \mathrm{BP}$ variability, or CVR, either under resting conditions, during elbow movement, or when combining both conditions.

\section{DISCUSSION}

In general, to assess the brain's circulatory system by TFA, TFA is applied to the BP-CBFV relationship. When CA is intact, the CBFV phase precedes the $\mathrm{BP}$ phase. When $\mathrm{CA}$ 

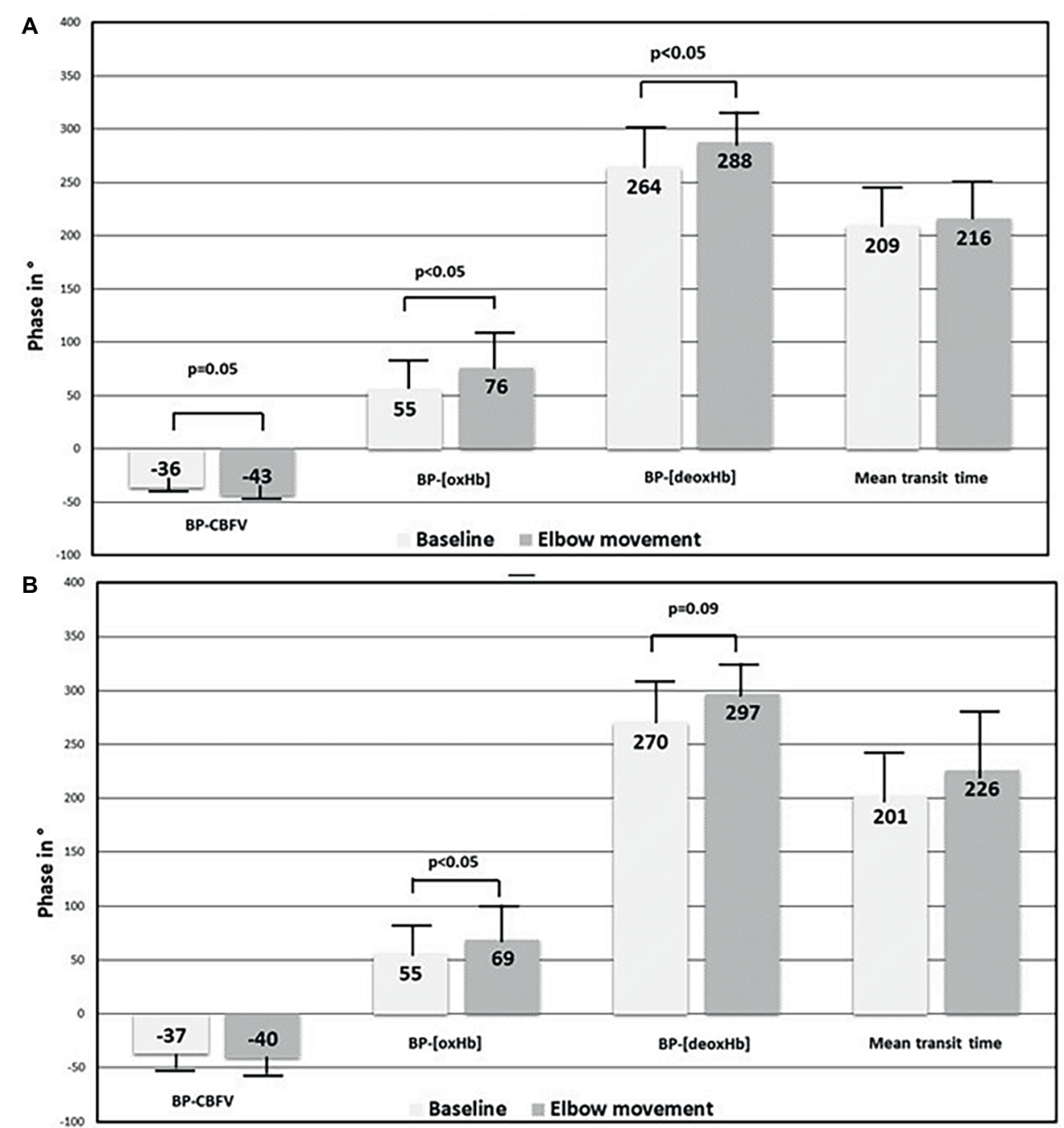

FIGURE 2 | Comparison of phase shift results between baseline at rest and elbow movement in macrocirculation and microcirculation. (A) Right side and (B) left side. BP, blood pressure; CBFV, cerebral blood flow velocity; [oxHb], oxygenated hemoglobin concentration; [deoxHb], deoxygenated hemoglobin concentration.

is disturbed, this phase lead becomes smaller. This change in phase lead is variable, but when CA fails (for example by increasing blood $\mathrm{CO}_{2}$ partial pressure), it increases as a result of vasodilation (or loss of vessel stiffness). Our baseline findings related to the BP-CBFV relationship are consistent with those of previously published literature (Meel-van den Abeelen et al., 2014; Claassen et al., 2016; Sanders et al., 2018). If the TFA approach is applied to the microcirculation parameters $[\mathrm{oxHb}]$ and [deoxHb] and related to $\mathrm{BP}$ or $\mathrm{CBFV}$ as the driving (input) parameter, our knowledge about the human microcirculation's dynamic remains limited. Gain varies widely, with inconsistent results. Under resting conditions, the differences in phase between $\mathrm{BP}$ or $\mathrm{CBFV}$ and [oxHb] or [deoxHb] range between 15 and $55^{\circ}$ for $\mathrm{BP}$-[oxHb], between 44 and $84^{\circ}$ for CBFV-[oxHb], between 209 and $265^{\circ}$ for BP-[deoxHb], and about $270^{\circ}$ for the CBFV-[deoxHb] relationship (Reinhard et al., 2006; Cheng et al., 2012;
Phillip et al., 2012; Fantini, 2014; Müller et al., 2016; Elting et al., 2018). The phase shift differences depend mostly on the mathematical approach used, data preparation, and the use of either spontaneous oscillatory CBF changes or enforced CBF changes by, for example, forced breathing (Reinhard et al., 2003, 2006; Cheng et al., 2012; Phillip et al., 2012; Müller et al., 2016; Elting et al., 2018). The reported mean transit times range between $190^{\circ}$ (corresponding to $5.15 \mathrm{~s}$ at $0.1 \mathrm{~Hz}$ ) in the investigation of Reinhard et al. (2006), $205^{\circ}$ in our present investigation (corresponding to $5.6 \mathrm{~s}$ ), and $120^{\circ}$ (corresponding to 3.2 s) in Elting et al. (2018). Although these transit time differences appear large, they are in the normal range when cerebral blood flow studies using positron emission tomography or dynamic susceptibility contrast-enhanced MRI are considered for comparisons. For cortical or hemispheric blood flow, the transit time is reported to vary between 2 and $8 \mathrm{~s}$, mainly around 4.3-5.1 s 
(Minns and Merrick, 1991; Ibaraki et al., 2007). Prolonged elbow movement led to several hemodynamic changes. It must be kept in mind that BP elevation and hypocapnia will lead to vasoconstriction (and an increased vessel stiffness) of the resistance vessels (Birch et al., 1995; Zhang et al., 1998, 2009; Panerai et al., 1999; Müller et al., 2003, 2019; Serrador et al., 2005), and a corresponding gain decrease in the TFA of the BP-CFBV relationship. In our work, the BP-CBFV relationship yielded a gain increase, indicating vasodilation despite vasoconstricting factors. Thus, from a macrocirculatory view, CBF increases at the macro- and the microcirculatory levels were induced by vasodilation, which would fit the increased metabolic demands. The demands were more prominent on the right side, explaining the stimulus-dependent side-to-side differences in [oxHb] (higher on the right side) and CVR (lower on the right side). At the microcirculatory level, forced breathing or hyperventilation increases phase shifting between BP and [oxHb] (Cheng et al., 2012). This finding is likely related to the macroangiopathic BP-CBFV changes in this condition (the BP-CBFV phase shift becomes more negative, meaning that $\mathrm{BP}$ is more delayed before entering intracranial circulation). Because our subjects hyperventilated, we consider our results to be consistent with those of Cheng et al. (2012), though [oxHb] may seem to respond more sensitively to $\mathrm{BP}$ changes than $\mathrm{CBFV}$ because phase changes were only marginally present in the BP-CBFV relationship.

Our study has limitations. Our CBF increases results are valid for movement tasks as the results are similar when movement is performed against a person's own arm's gravity or hand gripping at 30\% of a person's maximal hand gripping power (Caldas et al., 2018). Whether the dCA changes induced by movements are valid for changes in flow across the entire human cortex remains to be assessed since, for example, visual stimuli may provoke a different response pattern in the visual cortex compared to the motor cortex (Wolf et al., 2002). Further, our results are not independent of changes in $\mathrm{ETCO}_{2}$ and CVR. It appears necessary to at least control for $\mathrm{CO}_{2}$ changes in further studies. Nonetheless, the phase in the $\mathrm{BP}-[\mathrm{oxHb}]$ system seems more promising than in the BP-CBFV system for our intended purpose of grading VNC.

The variances as indicated by the SDs in the NIRS parameter-dependent systems were large and corresponded to the findings of Cheng et al. (2012). Further data processing with the help of filtering might be helpful in reducing variance

\section{REFERENCES}

Birch, A. A., Dirnhuber, M. J., Hartley-Davies, R., Iannotti, F., and Neil-Dwyer, G. (1995). Assessment of autoregulation by means of periodic changes in blood pressure. Stroke 26, 834-837. doi: 10.1161/01.STR.26.5.834

Birns, J., Jarosz, J., Markus, H. S., and Kalra, L. (2009). Cerebrovascular reactivity and dynamic autoregulation in ischaemic subcortical white matter disease. J. Neurol. Neurosurg. Psychiatry 80, 1093-1098. doi: 10.1136/jnnp.2009.174607

Caldas, J. R., Panerai, R. B., Salinet, A. M., Seng-Shu, E., Ferreira, G. S. R., Camara, L., et al. (2018). Dynamic cerebral autoregulation is impaired during
(Elting et al., 2018) but may lead to the loss of valuable information. Between-subject variability seems large and remains an unresolved problem (Sanders et al., 2018). One way to overcome subject variability is using a standardized examination setting and recording and reporting procedures (Claassen et al., 2016; Sanders et al., 2018). So far, our TFA results of the BP-CBFV system are consistent with those of a recent survey (Sanders et al., 2018). Another investigation could address whether using TF estimates as a strict linear method is adequate for assessing microcirculation. Aside from one study by Rowley et al. (2007), which discusses the presence of non-stationarity in the brain's slow oscillation, few studies address [oxHb]- and [deoxHb]-dependent systems with regard to the presence of nonlinearity or non-stationarity.

In conclusion, in our experiment assessing elbow movements performed against gravity, we found an increase in cerebral blood flow in macro- and microcirculation, which was accompanied by a small but significant BP increase and a small but significant $\mathrm{ETCO}_{2}$ decrease. Despite vasoconstricting effects of BP increase and ETCOE decrease, the increase in the BP-CBFV system indicates vasodilation. With $\mathrm{BP}$ oscillations as the reference, elbow movement induced a significant delay in [oxHb] and [deoxHb] oscillations while leaving the transit time between [oxHb] and [deoxHb] unchanged. Microcirculatory changes in phase seem to be more convenient than macrocirculation responses when using complex physiological processes for grading the response of VNC.

\section{DATA AVAILABILITY STATEMENT}

The datasets generated for this study are available on request to the corresponding author.

\section{ETHICS STATEMENT}

The studies involving human participants were reviewed and approved by Ethikkommission Nordwestschweiz. The patients/ participants provided their written informed consent to participate in this study.

\section{AUTHOR CONTRIBUTIONS}

Both authors contributed equally to the work. submaximal isometric handgrip in patients with heart failure. Am. J. Physiol Heart Circ. Physiol. 315, H254-H261. doi: 10.1152/ajpheart.00727.2017

Chance, B., Zhuang, Z., Un Ah, C., Alter, C., and Lipton, L. (1993). Cognitionactivated low-frequency modulation of light absorption in human brain. Proc. Natl. Acad. Sci. USA 90, 3770-3774.

Cheng, R., Shang, Y., Hayes, D. Jr., Saha, S. P., and Yu, G. (2012). Noninvasive optical evaluation of spontaneous low frequency oscillations in cerebral hemodynamics. NeuroImage 62, 1445-1454. doi: 10.1016/j.neuroimage.2012.05.069

Claassen, J. A., Meel-van den Abeelen, A. S., Simpson, D. M., and Panerai, R. B. International Cerebral Autoregulation Research Network (CARNet) (2016). Transfer function analysis of dynamic cerebral autoregulation: a white paper 
from the international cerebral autoregulation research network. J. Cereb. Blood Flow Metab. 36, 665-680. doi: 10.1177/0271678X15626425

de Heus, R. A. A., de Jong, D. L. K., Sanders, M. L., van Spijker, G. J., Oudegeest-Sander, M. H., Hopman, M. T., et al. (2018). Dynamic regulation of cerebral blood flow in patients with Alzheimer disease. Hypertension 72, 139-150. doi: 10.1161/HYPERTENSIONAHA.118.10900

Duncombe, J., Kitamura, A., Hase, Y., Ihara, M., Kalaria, R. N., and Horsburgh, K. (2017). Chronic cerebral hypoperfusion: a key mechanism leading to vascular cognitive impairment and dementia. Closing the translational gap between rodent models and human vascular cognitive impairment and dementia. Clin. Sci. 131, 2451-2468. doi: 10.1042/CS20160727

Elting, J. W. J., Tas, J., Aries, M. J. H., Czosnyka, M., and Maurits, N. M. (2018). Dynamic cerebral autoregulation estimates derived from near infrared spectroscopy and transcranial Doppler are similar after correction for transit time and blood flow and blood volume oscillations. J. Cereb. Blood Flow Metab. 38. doi: $10.1177 / 0271678 \times 18806107$ [Epub ahead of print].

Fantini, S. (2014). Dynamic model for the tissue concentration and oxygen saturation of hemoglobin in relation to blood volume, flow velocity, and oxygen consumption: implications for functional neuroimaging and coherent hemodynamics spectroscopy (CHS). NeuroImage 85, 202-221. doi: 10.1016/j. neuroimage.2013.03.065

Ibaraki, M., Ito, H., Shimosegawa, E., Toyoshima, H., Ishigame, K., Takahashi, K., et al. (2007). Cerebral vascular mean transit time in healthy humans: a comparative study with PET and dynamic susceptibility contrast-enhanced MRI. J. Cereb. Blood Flow Metab. 27, 404-413. doi: 10.1038/sj.jcbfm.9600337

Marmarelis, V. Z., Shin, D. C., Orme, M. E., and Zhang, R. (2014). Modelbased physiomarkers of cerebral hemodynamics in patients with mild cognitive impairment. Med. Eng. Phys. 36, 628-637. doi: 10.1016/j.medengphy.2014.02.025

Marmarelis, V. Z., Shin, D. C., Tarumi, T., and Zhang, R. (2017). Comparison of model-based indices of cerebral autoregulation and vasomotor reactivity using transcranial Doppler versus near-infrared spectroscopy in patients with amnestic mild cognitive impairment. J. Alzheimers Dis. 56, 89-105. doi: 10.3233/JAD-161004

Meel-van den Abeelen, A. S., Simpson, D. M., Wang, L. J., Slump, C. H., Zhang, R., Tarumi, T., et al. (2014). Between-centre variability in transfer function analysis, a widely used method for linear quantification of the dynamic pressure-flow relation: the CARNet study. Med. Eng. Phys. 36, 620-627. doi: 10.1016/j. medengphy.2014.02.002

Minns, R. A., and Merrick, M. V. (1991). "Cerebral perfusion pressure and nett cerebral mean transit time in childhood hydrocephalus" in Annual review of hydrocephalus. eds. S. Matsumoto, K. Sato, N. Tamaki, and S. Oi (Berlin, Heidelberg: Springer), 25-26.

Müller, M., Bianchi, O., Erülkü, S., Stock, C., Schwerdtfeger, K., and Homburg Traumatic Brain Injury Group (2003). Changes in linear dynamics of cerebrovascular system after severe traumatic brain injury. Stroke 34, 1197-1202. doi: 10.1161/01. STR.0000068409.81859.C5

Müller, M., Österreich, M., Müller, A., and Lygeros, L. (2016). Assessment of the brain's macro- and microcirculatory blood flow responses to $\mathrm{CO}_{2}$ via transfer function analysis. Front. Physiol. 7:162. doi: 10.3389/fphys.2016.00162

Müller, M., Österreich, M., von Hessling, A., and Smith, R. S. (2019). Incomplete recovery of cerebral blood flow dynamics in sufficiently treated high blood pressure. J. Hypertens. 37, 372-379. doi: 10.1097/HJH.0000000000001854

Murkin, J. M., and Arango, M. (2009). Near-infrared spectroscopy as an index of brain and tissue oxygenation. Br. J. Anaesth. 103(Suppl. 1), i3-i13. doi: 10.1093/bja/aep299

Obrig, H., Neufang, M., Wenzel, R., Kohl, M., Steinbrink, J., Einhäupl, K., et al. (2000). Spontaneous low frequency oscillations of cerebral hemodynamics and metabolism in human adults. NeuroImage 12, 623-639. doi: 10.1006/ nimg. 2000.0657
Panerai, R. B., Dawson, S. L., and Potter, J. F. (1999). Linear and nonlinear analysis of human dynamic cerebral autoregulation. Am. J. Phys. 277, H1089-H1099. doi: 10.1152/ajpheart.1999.277.3.H1089

Pantoni, L. (2010). Cerebral small vessel disease: from pathogenesis and clinical characteristics to therapeutic challenges. Lancet Neurol. 9, 689-701. doi: 10.1016/S1474-4422(10)70104-6

Phillip, D., Schytz, H. W., Selb, J., Payne, S., Iversen, H. K., Skovgaard, L. T., et al. (2012). Low frequency oscillations in cephalic vessels assessed by near infrared spectroscopy. Eur. J. Clin. Investig. 42, 1180-1188. doi: 10.1111/j.1365-2362.2012.02704.x

Reinhard, M., Müller, T., Guschlbauer, B., Timmer, J., and Hetzel, A. (2003). Transfer function analysis for clinical evaluation of dynamic cerebral autoregulation - a comparison between spontaneous and respiratory-induced oscillations. Physiol. Meas. 24, 27-43. doi: 10.1088/0967-3334/24/1/303

Reinhard, M., Wehrle-Wieland, E., Grabiak, D., Roth, M., Guschlbauer, B., Timmer, J., et al. (2006). Oscillatory cerebral hemodynamics-the macro- vs. microvascular level. J. Neurol. Sci. 250, 103-109. doi: 10.1016/j.jns.2006.07.011

Rowley, A. B., Payne, S. J., Tachtsidis, I., Ebden, M. J., Whiteley, J. P., Gavaghan, D. J., et al. (2007). Synchronization between arterial blood pressure and cerebral oxyhaemoglobin concentration investigated by wavelet crosscorrelation. Physiol. Meas. 28, 161-173. doi: 10.1088/0967-3334/28/2/005

Salinet, A. S. M., Robinson, T. G., and Panerai, R. B. (2013). Active, passive and motor imagery paradigms: component analysis to assess neurovascular coupling. J. Appl. Physiol. 114, 1406-1412. doi: 10.1152/japplphysiol.01448.2012

Salinet, A. S. M., Robinson, T. G., and Panerai, R. B. (2015). Effects of cerebral ischemia on human neurovascular coupling, $\mathrm{CO}_{2}$ reactivity, and dynamic cerebral autoregulation. J. Appl. Physiol. 118, 170-177. doi: 10.1152/ japplphysiol.00620.2014

Sanders, M. L., Claassen, J. A. H. R., Aries, M., Bor-Seng-Shu, E., Caicedo, A., Chacon, M., et al. (2018). Reproducibility of dynamic cerebral autoregulation parameters: a multi-Centre, multi-method study. Physiol. Meas. 39:125002. doi: 10.1088/1361-6579/aae9fd

Serrador, J. M., Sorond, F. A., Vyas, M., Gagnon, M., Iloputaife, I. D., and Lipsitz, L. A. (2005). Cerebral pressure-flow relations in hypertensive elderly humans: transfer gain in different frequency domains. J. Appl. Physiol. 98, 151-159. doi: 10.1152/japplphysiol.00471.2004

Wolf, M., Wolf, U., Toronov, V., Michalos, A., Paunescu, L. A., Choi, J. H., et al. (2002). Different time evolution of oxhemoglobin and deoxhemoglobin concentration changes in the visual and motor cortex during functional stimulation: a near-infrared spectroscopy study. NeuroImage 16, 704-712. doi: 10.1006/nimg.2002.1128

Zhang, R., Behbehani, K., and Levine, B. D. (2009). Dynamic pressure-flow relationship of the cerebral circulation during acute increase in arterial pressure. J. Physiol. 587, 2567-2577. doi: 10.1113/jphysiol.2008.168302

Zhang, R., Zuckerman, J. H., Giller, C. A., and Levine, B. D. (1998). Transfer function analysis of dynamic cerebral autoregulation in humans. Am. J. Phys. 274, H233-H241.

Conflict of Interest: The authors declare that the research was conducted in the absence of any commercial or financial relationships that could be construed as a potential conflict of interest.

Copyright (๑) 2019 Müller and Österreich. This is an open-access article distributed under the terms of the Creative Commons Attribution License (CC BY). The use, distribution or reproduction in other forums is permitted, provided the original author(s) and the copyright owner(s) are credited and that the original publication in this journal is cited, in accordance with accepted academic practice. No use, distribution or reproduction is permitted which does not comply with these terms. 